\title{
Derivaciones de la Observancia de la Participación Ciudadana y Perspectiva de Género en los Presupuesto de Egresos de un Municipio Sonorense 2012-2015
}

\author{
Adria Velia González Beltrones ${ }^{2}$ \\ Guadalupe Aleida Valenzuela Miranda ${ }^{3}$ \\ María Dolores Rocha Ontiveros ${ }^{4}$ \\ Jesús Miguel Maya Rodríguez ${ }^{5}$
}

Sumario: Introducción II. Primera Etapa III.Segunda Etapa IV. Tercera Etapa

V. Conclusiones

\section{Resumen}

En este documentos se plasman las derivaciones de la experiencia del proyecto de investigación-acción en Bacoachi, Municipio de Sonora quien apoyado por la Academia de Derecho Administrativo del Departamento de Derecho de la Universidad de Sonora implementó la metodología del Presupuesto Participativo con Perspectiva de género durante los ejercicios 2014 y 2015.Convirtiendose en el primer municipio en cumplir así con lo dispuesto en la Ley de Participación Ciudadana del Estado de Sonora, que entró en vigor en julio de 2011, y dentro de la cual se establece el presupuesto participativo, como una metodología a observar tanto para el gobierno del estado como para los 72 municipios de la entidad. Al respecto, cabe cuestionarse : ¿Cuáles son los alcances de la implementación de la metodología? ¿Cuántas personas con esta responsabilidad se capacitaron en cada dependencia del municipio de la experiencia ? ¿Cuáles son los beneficios de la formación que se ha ofrecido al respecto? Al elaborarse el presupuesto de egresos teniendo en cuenta la situación y posición de partida diferenciada de mujeres y hombres ¿Es previsible el impacto que puede tener su ejecución en unas y otros, para que contribuya a disminuir las desigualdades y a promover la igualdad? Son las respuestas a tales interrogantes ¿mediana o altamente satisfactorias?. ¿Cuales son la Condiciones necesarias para que se pueda replicar la experiencia?

Palabras clave: Presupuesto participativo, perspectiva de género

${ }^{1}$ Ponencia presentada en el $2^{\circ}$. Congreso de Investigación. Instituciones, Gobierno y Sociedad. COLSON,UNAM,SUJ, UAGTO, UANL,UNISON.Hermosillo, Sonora,21 y 22 de mayo de 2015.

${ }^{2}$ Docente investigadora de la DCS de la Universidad de Sonora, integrantes del CAEC 109-USON

${ }^{3}$ Docente investigadora de la DCS de la Universidad de Sonora, integrantes del CAEC 109-USON

${ }^{4}$ Docente investigadora de la DCS de la Universidad de Sonora, integrantes del CAEC 109-USON

${ }^{5}$ Estudiante de Doctorado en el COLSON 


\section{Summary: Introduction II. First stage III. Second stage IV. Third stage V. conclusions Abstract}

This document reflects the derivations of the experience of the action-research project in Protected, Municipality of Sonora who supported by the Academy of Administrative Law from the Department of Law at the University of Sonora implement the methodology of the Participatory Budget with gender perspective during the years 2014 and 2015.Becoming the first municipality in complying with the provisions of the Law on Citizen Participation of the State of Sonora, which entered into force in July 2011, and within which establishes the participatory budget, as a methodology to look at both the state government as to the 72 municipalities in the state. In regards to this, it may be questionable: What are the scope of the implementation of a methodology? How many people with this responsibility are trained in each unit of the municipality of the experience? What are the benefits of the training that has been offered in this regard? At the time of the preparation of the federal budget taking into account the situation and position of separate item of women and men do is predictable the impact that can have its implementation in some and others, to contribute to reducing inequalities and to promote equality? Are the answers to such questions medium or highly satisfactory? What are the necessary conditions to replicate the experience?

Key Words: Participatory budget, gender perspective.

\section{Introducción}

La Ley de Participación Ciudadana del Estado de Sonora (2011), establece dentro de los instrumentos de participación ciudadana, el presupuesto participativo, como una metodología a observar(obligación) tanto para el gobierno del estado como para los 72 municipios de la entidad. Por lo tanto, desde el 2012 se tuvo la posibilidad de formular los presupuestos municipales con base en la participación 
BIOLEX Revista Jurídica del Departamento de Derecho UNISON URC

Academia de Derecho Administrativo

Tercera Época Año 7. No 12 Enero-Junio de 2015

ciudadana (presupuesto participativo) ${ }^{6} \mathrm{y}$, vinculando dicho instrumento con las disposiciones de la Ley para la Igualdad entre Mujeres y Hombres en el Estado

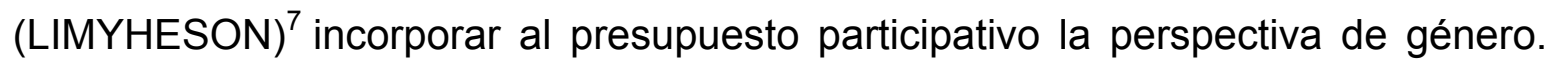
Sin embargo, de la información disponible en los portales de los municipios y de los boletines oficiales publicados que contienen los planes municipales de desarrollo encontramos que se hicieron con base en la consulta popular a que hace referencia la Ley de Planeación del Estado de Sonora, a través de los comités de planeación municipal (COPLAM); por lo tanto, si son con participación ciudadana; sin embargo, no incorporan la perspectiva de género que señala la Ley para la Igualdad entre mujeres y hombres del Estado de Sonora.

\section{Objetivo General}

Establecer las derivaciones de la experiencia del proyecto de investigaciónacción en Bacoachi, Municipio de Sonora quien apoyado por la Academia de

\footnotetext{
${ }^{6}$ De conformidad con la Ley de Participación Ciudadana, en su artículo 87, el presupuesto participativo tendrá por objeto: I.- Propiciar una distribución democrática de los recursos públicos de que disponen los gobiernos estatal y municipales, mediante un mecanismo público, objetivo, transparente y auditable, que posibilita intervenir en la solución de los problemas prioritarios de las comunidades sonorenses; II.- Efectuar obras prioritarias para la recuperación del espacio público; el mejoramiento y rehabilitación de calles; la rehabilitación o creación de áreas verdes; el mejoramiento o construcción de infraestructura cultural, deportiva y recreativa; así como acciones de desarrollo sustentable, fortalecimiento de la seguridad pública y la cultura;III.- Generar un proceso de democracia directa, voluntaria y universal, que contribuya a fortalecer espacios comunitarios de reflexión, análisis, revisión y solución a los problemas prioritarios, construyendo una ciudadanía consciente y participativa; y IV.- Establecer un vínculo corresponsable entre el gobierno y los gobernados que permita generar procesos ciudadanos de análisis, programación, vigilancia y control de los recursos públicos.

${ }^{7}$ El artículo14 de la Ley para la Igualdad entre Mujeres y Hombres del Estado de Sonora, dispone que corresponde a los ayuntamientos de los municipios: I.- Ejecutar la política municipal en materia de igualdad entre mujeres y hombres, en concordancia con las políticas nacional y estatal; II.Coadyuvar con el gobierno federal y con el gobierno estatal, en la consolidación de los programas en materia de igualdad entre mujeres y hombres; III.- Diseñar, formular y aplicar campañas de concientización que promuevan los valores y contenidos de la presente ley; IV.- Fomentar la participación social, política y ciudadana dirigida a lograr la igualdad entre mujeres y hombres, tanto en las áreas urbanas como en las rurales; y V.- Incorporar en sus Proyectos de Presupuestos de Egresos la asignación de recursos para el cumplimiento de la Política Municipal en Materia de Igualdad entre Mujeres y Hombres.
} 
Derecho Administrativo del Departamento de Derecho de la Universidad de Sonora implementó la metodología del Presupuesto Participativo con Perspectiva de Género ${ }^{8}$ durante los ejercicios 2014 y 2015.

\section{Primera Etapa}

La Academia de Derecho Administrativo ADA pretendió a través de la impartición del Seminario Presupuesto Participativo con Perspectiva de Género en municipios el objetivo de desarrollar y fortalecer las capacidades de los participantes para incorporar la participación ciudadana y la perspectiva de género en la elaboración del Plan Municipal de Desarrollo 2012-2015 y del presupuesto municipal de egresos 2013 y posteriores, atendiendo las disposiciones normativas correspondientes. En principio,los funcionarios participantes de los Ayuntamientos de los Municipios de Huepac, Hermosillo y Baviácora del Estado de Sonora para el periodo constitucional del 16 de septiembre de 2012 al 15 de septiembre de 2015 , se comprometieron a elaborar sus planes municipales de desarrollo y los presupuestos de egresos de manera participativa y con perspectiva de género, siguiendo la metodología aprendida. Paradójicamente, fue el Municipio de Bacoachi -que por por causas de fuerza mayor si bien no asistió al Seminario

\footnotetext{
${ }^{8}$ Los presupuestos con perspectiva de género son herramientas que buscan contribuir a la erradicación de las desigualdades existentes entre mujeres y hombres. Son previsiones de ingreso y gasto público, basadas en políticas y programas con orientaciones para la igualdad entre mujeres y hombres. Orientan los recursos públicos de modo que se distribuyan de acuerdo con las necesidades y los intereses específicos de cada grupo de población, concretamente de mujeres y hombres. Sus antecedentes jurídicos nacionales inmediatos PROIGUALDAD 2013-2018, derivado del Plan Nacional de Desarrollo PND: Alcanzar la igualdad sustantiva entre mujeres y hombres, en un contexto de democracia participativa, utilizando la planeación, programación y presupuesto con perspectiva de género. En el ámbito Federal, los avances importantes que deberían ser tomados como referencia para acciones locales: En el 2008 se incorporó ANEXO en presupuesto de egresos de la Federación: Erogaciones para la Igualdad entre Mujeres y Hombres ... \$ 7 mil millones; en 2014, diecinueve mil millones en más de 100 programas.
} 
BIOLEX Revista Jurídica del Departamento de Derecho UNISON URC

Academia de Derecho Administrativo

Tercera Época Año 7. No 12 Enero-Junio de 2015

impartido en noviembre de 2012, mostró desde principios del año $2013^{9}$ su interés en aprender la metodología para la elaboración del presupuesto participativo con perspectiva de género de la ADA y en coincidencia con SUMA ${ }^{10}$---quien a su vez facilitó la metodología para la instrumentación de iniciativas económicas para el empoderamiento económico de las mujeres del municipio de Bacoachi--simultáneamente se participó los días 13 y 14 de junio de 2013 con los Cursos Taller de antecedentes realizados en el municipio de Bacoachi, Sonora ${ }^{11}$. En Sesión el H. Cabildo de Bacoachi tomó el Acuerdo Número 02 Sesión Ordinaria, Acta Número 15 de Fecha 04 de julio de 2013 de aplicar el instrumento de participación ciudadana de Presupuesto Participativo con Perspectiva de género para el ejercicio fiscal 2014 en el presupuesto de Egresos .

\footnotetext{
${ }^{9}$ Proporcionando la información municipal requerida por la Academia para su debida orientación.

${ }^{10}$ SUMA, Transformando para la Igualdad, es resultado de la convocatoria de propuestas lanzada en 2009 por el Fondo para la Igualdad de Género de ONU Mujeres a nivel mundial para apoyar proyectos para el fortalecimiento político y económico de las mujeres. En junio de 2010 se dieron a conocer los resultados: quince países del mundo resultaron ganadores, tres de América Latina y entre ellos nuestro proyecto. En este contexto, el proyecto SUMA cuenta con el aval del Instituto Nacional de las Mujeres y la recomendación del Instituto Federal Electoral y del Tribunal Electoral del Poder Judicial de la Federación.SUMA lo conforman las siguientes organizaciones sociales: Equidad de Género; Ciudadanía, Trabajo y Familia; Instituto de Liderazgo Simone de Beauvoir; Mujeres Trabajadoras Unidas; Inclusión Ciudadana y Liderazgo, Gestión y Nueva Política. SUMA, busca la efectiva implementación de la Ley General para la Igualdad entre Mujeres y Hombres 2006 y del Código Federal para las Instituciones y Procesos Electorales (COFIPE), para coadyuvar en la participación y representación política equilibrada de las mujeres y los hombres y al logro de la igualdad y equidad en la vida económica nacional.

${ }^{11}$ uno de los seis municipios seleccionados por Iniciativa SUMA para propiciar en ellos un “cambio estructural para la Igualdad, mediante "una visión integrada del desarrollo", con la metodología de la Comisión Económica para América Latina CEPAL-- consistente en seguir una ruta que conjuga tres dimensiones del desarrollo económico y social: Cambio Estructural, Convergencia Tecnológica, Promoción de la Igualdad--(Para cada dimensión se trabaja sobre criterios que permiten determinar tanto los aspectos críticos, como las condiciones desfavorables y favorables que tienen los municipios).
} 


\section{Segunda Etapa}

En el marco del Proyecto, con fecha 9 de septiembre de 2013, acudimos a Bacoachi, Sonora, a invitación de la Presidenta Municipal para asistir a la instalación del Comité de Planeación Municipal. Instituciones participantes: Universidad de Sonora a través de la Academia de Derecho Administrativo e Iniciativa SUMA.Lugar de intervención: Palacio Municipal del Municipio de Bacoachi, Sonora. Actividades realizadas: Instalación del COPLAM del Municipio de Bacoachi, Sonora.

\section{Contexto}

Como parte de los compromisos del H. Ayuntamiento de Bacoachi, la Presidenta Municipal María Enedina Díaz González instaló formalmente la sesión del Comité de Planeación Municipal, previa convocatoria e invitaciones. ${ }^{12}$

\section{Acciones}

Con la participación de la Presidenta Municipal, se realizaron las siguientes acciones e intervenciones:

Se planteó el objeto de la reunión, siendo este el cumplimiento de las disposiciones de la Ley de Participación Ciudadana y la Ley para la Igualdad entre Mujeres y Hombres del Estado de Sonora y, en especial, que para el ejercicio presupuestal 2014, el municipio cuente con un presupuesto participativo, y además considere la perspectiva

\footnotetext{
${ }^{12}$ Se realizó la reunión con la presencia de los integrantes el Cabildo, de las áreas de la administración pública municipal y de los representantes de los diversos sectores de la sociedad y de la Delegación de la Sagarpa. Por la Universidad de Sonora, asistieron las doctoras María Inés Aragón Salcido, Adria Velia González Beltrones y M.I.E. Jesús Miguel Maya Rodríguez.
} 
de género para su elaboración la participación de todos(as) los(as) sectores de la sociedad del Municipio de Bacoachi en el proceso de programación, presupuestación, control y evaluación de las actividades para el desarrollo del municipio, de conformidad con el Plan Municipal de Desarrollo.

Se Informó que hay un calendario que tienen que aplicar para la elaboración de la Iniciativa de Ley de Ingresos para el ejercicio fiscal y Presupuesto de ingresos del ejercicio fiscal de 2014 y su presentación al Congreso del Estado, siendo esta fecha los primeros días de noviembre. $Y$, una vez que hayan aprobado la Ley de Ingresos, presentará al Ayuntamiento el Presupuesto de Egresos para el ejercicio fiscal de 2014, en el cual ya están trabajando, de acuerdo con el manual de programación que les proporciona el CEDEMUN ${ }^{13}$.

Que por ello, ha iniciado el proceso de programación en el seno del COPLAM, ya que es el órgano que debe conocer el proceso de consulta ciudadana para la elaboración del presupuesto participativo. Si bien tienen tiempo para desarrollar esta etapa de consulta con la ciudadanía, también lo es que deben iniciarse los trabajos. Lo novedoso es, el presupuesto participativo incorpora la perspectiva de género que pretenden impulsar desde el COPLAM.

Se otorgó el uso de la voz a las personas presentes, manifestándose como ideas generales las siguientes:

Por parte de las (os) académicas(os), hicieron referencia a que el ejercicio consiste en la participación de todas las personas del municipio, para la elaboración del presupuesto, recabando sus necesidades específicas e implementar el presupuesto con esas inquietudes. Concretando por la parte del género, que conviene

1. ${ }^{13}$ Hasta la fecha el Manual de Programación y Presupuestacion 2015 de CEDEMUN no incluye disposicion alguna para distinguir recursos enfocados a acciones afirmativas para la igualdad entre mujeres y hombres. 
dirigirlo hacia la implicación de la igualdad de oportunidades y derechos entre hombres y mujeres para alcanzar el desarrollo, buscando que en el Programa Operativo Anual, en la descripción de metas se desagregue la información por mujeres y hombres, para medir cuántas mujeres resultan beneficiadas con los programas. Igualmente, identificar los programas que benefician y disminuyen las brechas de desigualdad entre mujeres y hombres.

La Presidenta Municipal, en su carácter del Presidenta del COPLAM invitó a las (os) presentes a que manifestaran sus inquietudes que desde sus distintos sectores conocen, explicándoles que es una experiencia nueva, lo del presupuesto participativo, y además, que incorporar la perspectiva de género en el presupuesto vendrá a consolidar los apoyos que han venido entregando a diversas mujeres para resolver sus problemas económicos. Y, sobre todo apoyar en la capacitación para que las mujeres que quieran participar en lo futuro lo hagan en proyectos que sean rentables y el municipio las apoye en la gestión a realizar ante las instancias federales para que obtengan los recursos de los distintos Fondos que al efecto administran.

El Representante ejidal manifestó que consideraba que la representación ante el poder municipal la tienen a través de los regidores, los cuales en sesión de cabildo ejercen una representación indirecta del sentir de la comunidad, por lo cual confían en ellos (as) para que manifiesten las necesidades más apremiantes de la comunidad.

Uno de los Regidores del municipio, manifiesta que una de las problemáticas la representa la atención al autobús escolar, y esta ha sido atendida desde el frente que representa, e igual muestra disposición para aceptar cualquier invitación a asistir proveniente de alguno de los ejidos del Municipio de Bacoachi para recabar las propuestas y traerlas al órgano de gobierno municipal cuando así disponga la población. También, señalaron que se les están 
presentando una serie de problemas en el Registro Público de la Propiedad de Cananea porque no les quieren inscribir los títulos que ha expedido el ayuntamiento, en virtud de que hay problemas con el fundo legal de BACOACHI. La Dra. Aragón les informó que el fundo legal está sobre terrenos comunales y que ya la Presidenta Municipal está tramitando en la CORETT apoyo para resolver el problema que data desde hace muchos años. Uno de los asistentes a la reunión precitada ex presidente municipal, comentó que es un problema muy viejo, que es importante resolver de manera inmediata, pues es muy importante para todos, ya que les afecta no tener los títulos en regla.

La C. Presidenta Municipal, reconoce el trabajo de las (os) regidoras(es), del Síndico y de igual manera de personal que labora en la administración, la mayoría mujeres, y la importancia del Presupuesto participativo como un instrumento de participación en el Comité de Planeación Municipal, que viene a coadyuvar con la participación directa de las opiniones del pueblo, y sus prioridades, ante una realidad de muchas necesidades, y escasos recursos. El Regidor, manifestó que valora la opinión del representante ejidal, pero igual es importante que como $\mathrm{H}$. Ayuntamiento acerquen el gobierno a la manifestación directa de las necesidades específicas de la población.La C. Presidenta Municipal, invitó a la reunión del COPLAM a las dependencias federales y estatales, que tienen programas para los municipios, ya que es importante plasmar las ideas e inquietudes que se discutan en esta reunión e informarles a las autoridades. Se disculparon los delegados de CORETT, SEDATU y SEDESOL.Destacó la presencia de personal de la Delegación regional de SAGARPA, con sede en Cananea, y les agradeció su presencia, quienes manifestaron que era la primera invitación que les hacían en un proceso de participación ciudadana desde la base municipal y que definitivamente la planeación debe empezar por escuchar a la ciudadanía y que en su dependencia hay 
programas en los cuales la intervención de la mujer es prioritaria, las jóvenes y las indígenas y que ofrecían su apoyo al municipio para capacitación en los programas que ellos tenían.Comenta un participante que hay muchos programas gubernamentales no solo de SAGARPA, pero el desconocimiento de las reglas de operación para bajar esos recursos dificulta acceder a ellos, y también se requiere capacitación de las(os) funcionarias(os) públicos para la atención de las necesidades que enfrenta el municipio. Informa la Presidenta Municipal, que terminando la reunión del COPLAM, el personal de SAGARPA impartirá un curso Taller para elaborar proyectos a 60 mujeres invitadas y que tiene en interés en prepararse para elaborar proyectos productivos que mejoren su economía familiar.

Se realizaron los siguientes compromisos:

\section{Por parte de la Administración Municipal:}

Procesar la información obtenida de COPLAM y otras sesiones para la elaboración del Presupuesto Participativo con Perspectiva de Género.

Sostener reuniones de seguimiento de los compromisos con Iniciativa SUMA y la Academia de Derecho Administrativo.

\section{Por parte de la Academia de Derecho Administrativo:}

Continuar con el apoyo de la ejecución de la metodología del Presupuesto Participativo con Perspectiva de género.

Realizar próximas visitas de seguimiento de los objetivos y capacitación adicional que se requiera.

En este contexto, la presidenta Municipal asistió y participó, a invitación de Iniciativa Suma, en la II Cumbre Iberoamericana de Agendas Locales de Género, celebrada en Aguas Calientes, del 23 al 27 de septiembre de 2013, participando, junto con otras presidentas municipales, en la conferencia abierta el día 24 de 
BIOLEX Revista Jurídica del Departamento de Derecho UNISON URC Academia de Derecho Administrativo

Tercera Época Año 7. No 12 Enero-Junio de 2015

septiembre, en la mesa coordinada por Patricia Mercado: "Los municipios. El espacio para el empoderamiento económico de las mujeres. la experiencia de Iniciativa Suma, México." ${ }^{14}$.La presidenta Municipal después de los dos talleres impartidos por personal de SAGARPA y las orientaciones del INAES, revisó junto con su equipo, las opciones a presentar por las mujeres asisentes a la capacitación y asesoradas debidamente para la elaboración de sus proyectos a financiar por un Fondo Municipal a instituir en el Presupuesto de Egresos para el ejercicio fiscal 2014, como un anexo para apoyar proyectos que contribuyan a empoderar mujeres. Además, de incluir en el Programa Operativo anual 2014, en la estrategia programática municipal en el apartado de objetivos, estrategias y líneas de acción, apoyar proyectos productivos que contribuyan al empoderamiento de las mujeres en el municipio de Bacoachi. ${ }^{15}$ Una vez aprobado

\footnotetext{
${ }^{14}$ Igualmente, participó en la constitución de la Red Iberoamericana de Municipios por la Igualdad de Género e hizo la propuesta para que las doctoras María Inés Aragón Salcido y Adria Velia González Beltrones, en su carácter de Asesoras Académicas del municipio de Bacoachi y a la Iniciativa SUMA, cuya presidenta es la Lic. Patricia Mercado Castro, formaran parte de la citada Red Iberoamericana de Municipios por la Igualdad de Género. En la búsqueda de proyectos de empoderamiento económico para las mujeres del Municipio de Bacoachi, se asistió a las oficinas de INAES, en Hermosillo, donde se explicó que a principios de año, se abren las convocatorias para participar y que hay un Fondo para mujeres, ofreciendo asesoría y capacitación para explicar las reglas de operación.En Octubre de 2013 la Presidenta envió oficio al Director General del INAES, solicitando la cantidad de $\$ 100,000.00$ (Pesos Moneda Nacional) que será igualada con una cantidad similar por parte del Ayuntamiento para constituir un Fondo Municipal para financiar microempresas, fundamentalmente destinada a mujeres.Con fecha 29 y 30 de octubre de 2013 participaron, tanto la Presidenta Municipal de Bacoachi como las doctoras María Inés Aragón Salcido,Adria Velia González Beltrones y Aleida Guadalupe Valenzuela Miranda en el "Curso Taller Presupuestos Públicos", impartido por Iniciativa Suma, INMUJERES, ONUMUJERES, la Comisión de Equidad y Asuntos de Género del Congreso del Estado de Sonora y el Instituto Sonorense de la Mujer, en la ciudad de Hermosillo, Sonora

${ }^{15}$ Aunque el presupuesto total que se asignó al municipio es reducido, las acciones se encaminan a la agenda de género como las siguientes :Fondo de becas Educativas, son recursos para becas otorgados por el Gobierno Municipal para 50\% estudiantes mujeres y 37 hijos de Jefas de Familia.Aportaciones al Comercio, son recursos otorgados también por el Gobierno Municipal, para Proyectos Productivos para mujeres y/o mixtos donde la mayoría sean mujeres.Desde el inicio de la presente administración se esta apoyando a niñas y niños con capacidades diferentes de los cuales el $75 \%$ son mujeres. Se han generado también acciones de capacitación para el trabajo y apoyado 20 proyectos de autoempleo que elevan la autoestima de las mujeres y les permiten
} 
por el Congreso del Estado la Ley de Ingresos se celebró una reunión de COPLAM para aprobar los proyectos a incorporar al Presupuesto de Egresos de 2014 de corte participativo con perspectiva de género.

\section{Tercera Etapa}

En el marco del Proyecto, y en términos de la consulta para la elaboración de la Plataforma Electoral 2015, convocada por la FUNDACIÓN COLOSIO SONORA, en la mesa temática: Política y Gobierno, en el TEMA 2. Gobierno y Participacion Social: Marco legal de la participación social y ciudadana. Gobierno y redes sociales. Participación en la planeación, instrumentación, implementación, control y evaluación en los programas y proyectos gubernamentales. Vinculación y alianzas estratégicas del gobierno con instituciones de educación superior y centros de investigación, consideramos importante plantear la necesidad de que los próximos planes y programas estatal y municipales de desarrollo 2015 y los presupuestos de egresos se elaboren con base en el principio de la igualdad de derechos entre mujeres y hombres y la perspectiva de género. ${ }^{16}$

integrarse a actividades productivas, además de otorgar 22 becas a estudiantes universitarias, de igual forma estamos becando a 37 hijos de 27 madres jefas de familia cubriendo el $100 \%$ de este sector con dicho beneficio.

${ }^{16}$ Partimos de los antecedentes, con un repaso del marco internacional y el nacional, en especial revisando, las experiencias de los fondos y programas que se han instrumentado hasta llegar al Plan nacional de Desarrollo 2013-2018, que incorpora por primera vez en México la perspectiva de género $y$, en particular, el programa especial Proigualdad 2013-2018. En una segunda parte, examinamos el marco jurídico estatal, sobre la planeación y los presupuestos participativos con efectos vinculantes y las experiencias que nos evidencian que en Sonora en los instrumentos de planeación vigentes: planes estatal y municipales de desarrollo no se incorpora la perspectiva de género y que en forma incipiente se ha iniciado la experiencia municipal del presupuesto participativo con perspectiva de género, en el municipio de Bacoachi. 
Por todo ello, se presentaron las propuestas de reformas a varios ordenamientos $^{17}$, a fin de que en el año próximo (2015) se hagan las reformas que institucionalicen la perspectiva de género en la planeación democrática en el estado de Sonora, porque consideramos que se deben

“..diseñar políticas públicas de corto, mediano y largo plazo dirigidas a combatir las causas históricas y estructurales que impiden y obstaculizan su desarrollo al limitar, segregar, discriminar o excluir a las mujeres en muy diversos ámbitos, y con ello la participación de más de la mitad de la población mexicana en el desarrollo". ${ }^{18}$

$\mathrm{Y}$, porque

"debemos de impulsar la igualdad sustantiva entre mujeres y hombres, para que México alcance su máximo potencial, como semana el PND. "Sólo así el país podrá aspirar a ser un país democrático y participativo, y será posible alcanzar las cinco metas nacionales que el Gobierno de la República se ha propuesto: la de un México en Paz, un México Incluyente, un México con Educación de Calidad, un México Próspero y un México con Responsabilidad Global. Para ello, la contribución de las mujeres en todos los ámbitos, sin discriminación y bajo el eje rector de la igualdad sustantiva, es indispensable". ${ }^{19}$

Con fecha 6 de junio de 2014 se participó en el Foro de Mujeres en la Mesa:

Presupuestos con Perspectiva de Género en donde se destacó la importancia del proceso de planeación con perspectiva de género para el fortalecimiento de la participación ciudadana, y se procedió a formular las preguntas detonadoras ${ }^{20}$

\footnotetext{
17 a la Ley de Planeación del Estado de Sonora, a La Ley del Presupuesto de Egresos, Contabilidad Gubernamental y Gasto Público Estatal y a la de Gobierno y Administracion Municipal, en materia de presupuestos participativos con enfoque de género, para ser considerada en la plataforma.

${ }^{18}$ Programa Nacional para la Igualdad de Oportunidades y no Discriminación contra las Mujeres. PROIGUALDAD 2013-2018.D.O.F.30/08/2013

${ }^{19}$ Idem

20 1.- ¿Cuál es el estado que guardan los presupuestos con perspectiva de género, en sus diversas manifestaciones estatal y municipal?2.- ¿Cuál es el impacto que han tenido en la legislación de Sonora?

3.- ¿Qué acciones deberían emprender los siguientes actores: Federación, Estado, Municipio?
} 
Tercera Época Año 7. No 12 Enero-Junio de 2015

para que las participantes en la mesa reflexionaran sobre las mismas e hicieran las propuestas conducentes. En un segundo tiempo se dio cuenta con diversas propuestas con ponencias y propuestas por escrito ${ }^{21}$ Después de escuchar a los ponentes, se pasó a la sección de preguntas y respuestas de las cuales destaca entre otras la de "promover la adopción de presupuestos etiquetados para la igualdad de género en los gobiernos municipales y del Edo. de Sonora"

En Acta de Cabildo Número 44 de Sesión Ordinaria Celebrada en el Salón de Cabildo del Palacio Municipal, el día 04 de Diciembre del año 2014, se deliberó y aprobó por unanimidad de los integrantes de Cabildo, el Acuerdo No. Dos por el que el H. Ayuntamiento de Bacoachi, Sonora aprueba por unanimidad la aplicación del instrumento de participación ciudadana de presupuesto participativo, con perspectiva de género para el ejercicio fiscal 2015, el cual se llevará a cabo en el seno del Comité de Planeación Municipal (COPLAM).

En resumen, la respuesta a las 3 primeras siguientes interrogantes ¿Cuáles son los alcances de la implementación de la metodología? ¿Cuántas personas con esta responsabilidad se capacitaron en cada dependencia del municipio de la experiencia ? ¿Cuáles son los beneficios de la formación que se ha ofrecido al respecto? es:

R1.-Los alcances son múltiples puesto que la experiencia de la implementación

21 Grupo de participación social, un elemento ciudadano de socialización con perspectiva de género en colonias y localidades de sonora, presentado por Ana Luis Valdés.2.- Una propuesta del Instituto Sonorense de Admón. Pública (ISAP) de lo que se tiene que hacer para tener el presupuesto con perspectiva de género en los gobiernos municipales y el del estado de sonora, Ing. Alberto Hazz.3.- Experiencia en la elaboración del presupuesto de egresos participativo con perspectiva de género del municipio de Bacoachi, sonora. presentado por la Academia de Derecho Admvo. de la UNISON: Adria Velia Gonzalez, María Inés Aragón Salcido, María Dolores Rocha Ontiveros, Guadalupe Aleida Valenzuela Miranda, Alberto Hazz Díaz y Jesús Miguel Maya Rodríguez.4.- Avances de la experiencia en la elaboración del presupuesto de egresos participativo con perspectiva de género del municipio de Bacoachi, Sonora,por la presidenta municipal de Bacoachi, María Enedina Díaz. 
del presupuesto de egresos con perspectiva de género en el municipio de Bacoachi por dos años consecutivos es exitosa y se cumplió con la expectativa de cumplir con lo dispuesto en los ordenamientos jurídicos aplicables en tiempo y forma. Asimismo, con la metodología para el presupuesto participativo con perspectiva de género, se instrumentaron iniciativas económicas del nivel municipal para el empoderamiento económico de las mujeres de Bacoachi.

R2. Los integrantes del H Ayuntamiento de Bacoachi : 5 regidores, la presidenta Municipal, un Síndico Municipal, los titulares de Secretaría Municipal,Tesorerería,Contraloría, los titulares de las Direcciones de Servicios Públicos, de Seguridad Pública, de Obras Públicas, los Delegados de Unamichi y de Mututicachi.

R3. Que en todas las dependencias donde se elaboren presupuestos al integrarlos a los presupuestos generales de ingresos y de egresos con participación y perspectiva de género: se tomen en cuenta las necesidades específicas de hombres y mujeres, se tracen estrategias y líneas de acción para beneficiar a mujeres y hombres en los diferentes programas del $\mathrm{H}$ Ayuntamiento, se establezcan indicadores para conocer el impacto recibido por los hombres y/ o mujeres de Bacoachi.

R4.El nivel de participación de las mujeres y hombres de Bacoachi en el proceso de diseño, monitoreo e implementación de los Presupuestos Participativos con Perspectiva de Género es directa- interna, horizontal-vertical y transversal (la de las funcionarias y los funcionarios de la administración pública e integrantes del $\mathrm{H}$ Ayuntamiento); directa e indirecta, externa -interna, horizontal-vertical y 
transversal (la de los integrantes del COPLAM).

La respuesta a las últimas tres cuestiones: Al elaborarse el presupuesto de egresos teniendo en cuenta la situación y posición de partida diferenciada de mujeres y hombres, ¿Es previsible el impacto que puede tener su ejecución en unas y otros, para que contribuya a disminuir las desigualdades y a promover la igualdad?, Son las respuestas a tales interrogantes ¿mediana o altamente satisfactorias? y ¿Cuales son la Condiciones necesarias para que se pueda replicar la experiencia?, se lista a continuación:

R4.Si es previsible el impacto que puede tener su ejecución en unas y otros, para que contribuya a disminuir las desigualdades y a promover la igualdad porque previamente se implementó la Perspectiva de Género en la planeación, programación y presupuestación municipal, al elaborar o revisar el diagnóstico, al identificar inequidades, al definir el programa y sus componentes (marco lógico), al priorizar acciones, al diseñar o determinar indicadores, al determinar los recursos necesarios, al calcular el importe y ajuste a posibilidades, al establecer el tablero para su seguimiento, y al darle seguimiento para evaluar y continuar o en su caso corregir (retroalimentación).

R5. En el caso que nos ocupa las respuestas son altamente satisfactorias porque es el único municipio del Estado de Sonora que ha cumplido(ejercicios fiscales 2014 y 2015) con la normatividad aplicable en materia de participación y perspectiva de género en la planeación, programación y presupuestación municipal. Y medianamente satisfactorio, porque el avance en obtener 
integralmente los beneficios de la herramienta presupuestal puede aumentarse cada año y de esa manera beneficiar a mayor número de mujeres y hombres según las necesidades detectadas en el diagnóstico.

R.6 Voluntad política, ejercicio de funciones y participación ciudadana para exigir y en su caso entablar responsabilidades a quienes conforme a la normatividad están obligados a cumplir conforme lo indica sus preceptos y no lo hacen. En otras palabras, atender y dar cumplimiento al marco normativo que obliga a la aplicación de la perspectiva de género en los presupuestos estatal y municipales; dar cumplimiento a las disposiciones para el presupuesto participativo derivado de la Ley de Participación Ciudadana, mayor atención del Congreso Local para trabajar en colaboración con las administraciones públicas local y municipal en elaborar y hacer que fluyan a tiempo y en forma los dispositivos jurídicos referentes a los Presupuestos participativos con perspectiva de género.

A manera de Conclusiones derivadas de la observancia de la participación ciudadana y perspectiva de género en los presupuesto de egresos del Municipio Sonorensede Bacoachi 2012-2015:

Para atender y dar cumplimiento al marco normativo que obliga a la aplicación de la perspectiva de género en los presupuestos estatal y municipales del Estado de Sonora se requiere:

1. Capacitar a la totalidad del personal del sector público estatal y de los restantes 71 municipios en relación a los fundamentos y conceptos 
inherentes a la igualdad sustantiva entre mujeres y hombres,

2. Promover la adopción de presupuestos etiquetados para la igualdad de género en los gobiernos municipales y el del Estado de Sonora;

3. Que el Ejecutivo del Estado,en el Manual de Programación y Presupuestación 2015 incluya la disposicion para distribuir recursos enfocados a acciones afirmativas para la igualdad entre mujeres y hombres;

4. Que en el Manual de Programación y Presupuestación 2015 el CEDEMUN incluya la disposicion para distribuir recursos enfocados a acciones afirmativas para la igualdad entre mujeres y hombres;

5. Que en las orientaciones que emita el ISAF a los Ayuntamientos incluya la recomendación de distibuir recursos enfocados a acciones afirmativas para la igualdad entre mujeres y hombres;

6. Que el Congreso local verifique la incorporación del anexo presupuestario 2015 para la igualdad entre mujeres y hombres, que también los ayuntamientos incorporen anexos en sus respectivos ambitos presupuestarios;

7. Que estos recursos etiquetados para la igualdad entre mujeres y hombres se establezcan con normas que determinen que serán de la siguiente forma: Intransferibles,Irreductibles y Progresivos;

8. Aprovechar la asesoría y capacitación que ofrecen diversas instituciones de Sonora en la administración pública(Poder Ejecutivo) y en los otros dos Poderes;

9. Que los procesos de programación y presupuestación municipales se realicen aplicando la participación ciudadana con enfoque de género 
orientado a corregir desigualdades de oportunidad entre mujeres y hombres;

10. Que cada dependencia, entidad y órgano público y de cada municipio se cuente con personal competente en relación al uso de metodologías para la programación y presupuestación con perspectiva degénero vinculada al esquema del presupuesto por resultados;

11.Reformar las leyes de Planeación, Ley de Gobierno y Administración Municipal, Ley Orgánica del Poder Ejecutivo, Ley de Presupuesto de Egresos, Contabilidad Y Gasto Público, y La Ley del Instituto Superior de Auditoría Fiscal ISAF, para que se establezca la perspectiva de género como obligatoria en los Planes y Programas de Desarrollo Estatal y Municipal.

\section{Bibliografía y Webgrafía}

Aragón Salcido María Inés, Adria Velia González Beltrones et al."La perspectiva de género en el presupuesto participativo en el ámbito municipal en el Estado de Sonora: ¿Potestativa u Obligatoria?, presentada por: Adria Velia González Beltrones, María Inés Aragón Salcido, María Dolores Rocha Ontiveros, Guadalupe Aleida Valenzuela Miranda, en el IV congreso Iberoamericano y VI Congreso Mexicano de derecho administrativo, celebrado del 29 al 31 de octubre de 2012, en la Facultad de Derecho de la Universidad Veracruzana en la ciudad de Jalapa, Veracruz.

Aguilar, Lorena, Itzá Castañeda, Hilda Salazar y Jacqueline Siles, En búsqueda del género perdido. Equidad en áreas protegidas, Unión Mundial para la Naturaleza/Absoluto, San José Costa Rica, 2002.

Beltrán, Elena y Virginia Maquieira (eds.), Feminismos: Debates teóricos contemporáneos, Alianza Editorial, Madrid, 2001.

Budlender, Debie y Sharp Rhonda, Cómo realizar un análisis de presupuesto sensible al género. Secretaría de La Mancomunidad Británica.2000. [Disponible

en: http://presupuestoygenero.net/Herramientas/H028BudlenderGL.pdf]

Buscarons, Mónica, Presupuesto Participativo en Perspectiva de Género de Santa Cristina D'aro", Técnica de políticas de igualdad y coordinadora del servicio de información y atención a las mujeres (SIAD) del Ayuntamiento de Santa Cristina d'Aro, Cataluña, España, 2008. 
Cámara de Diputados, LX Legislatura, Foro Nacional de Presupuestos para la lgualdad entre Mujeres y Hombres. Centro de Estudios para el Adelanto de las Mujeres y la equidad de Género. H.Congreso de la Unión, México, 1ª.ed. 2007. Convención sobre la Eliminación de Todas las Formas de Discriminación contra la Mujer (CEDAW). Compilación sobre los principales Instrumentos Internacionales sobre Derechos Humanos de las Mujeres, Instituto Nacional de las Mujeres, 3ra. Edición. México, 2006..

Elson, Diane.Seguimiento de los Presupuestos Gubernamentales para el Cumplimiento de la CEDAW.InformeparaUNIFEM. Department of Sociology, University of Essex, UK/ Levy 2005,Economic Institute, Bard College, USA.

García, Brígida, "Empoderamiento y autonomía de las mujeres en la investigación sociodemográfica actual", en Revista Estudios Demográficos y Urbanos, núm.53, El Colegio de México, México, 2003.

Glosario de términos relacionados con la transversalidad de género, [en línea], España, Proyecto Equal 2007 [consultado el 26 de septiembre de 2012] en:http://www.eculturas.com/final/Documenta/texto/GlosarioGenero.pdf

Instituto Nacional par las Mujeres INMUJERES Desarrollo Local, con Igualdad de Género, Volumen V, Guía Conceptual, Instituto Nacional de las Mujeres, México, 2009

\section{Legislación}

"Ley de Gobierno y Administración Municipal", [Disponible en:

http://www.cgeson.gob.mx/servicios/leyes/estatal/leyes/Ley\%20075\%20De\%20Go b\%20y\%20Admon\%20Municipal.pdf]

"Ley de Participación Ciudadana del Estado de Sonora"[Disponible en: http://gobierno.sonora.gob.mx/documentos/

"Ley para la Igualdad entre Mujeres y Hombres en Sonora", [Disponible en:http://cedoc.inmujeres.gob.mx/ftpg/Sonora/son02.pdf

"Ley de Planeación para el Estado de Sonora", [Disponible en:

http://transparencia.esonora.gob.mx/

"Reglamento del COPLADES", [Disponible en:

http://docs.google.com/leaf?id=

"Ley de presupuesto de egresos, contabilidad gubernamental y gasto público del Estado de Sonora", [Disponible en: http://www.secsonora.gob.mx/ocda/uploads/wfd 